\title{
Serosurvey of Human Antibodies Recognizing Aedes aegypti D7 Salivary Proteins in Colombia
}

OPEN ACCESS

Edited by:

Ahmed Mohamed,

North Carolina State

University, United States

Reviewed by:

Keyue Ding,

Queen's University,

Canada

Xiangzhu Zhu,

Vanderbilt University,

United States

Ajit Kumar Karna,

New Mexico State

University, United States

${ }^{*}$ Correspondence:

Majid Jaberi-Douraki

jaberi@k-state.edu;

Tonya M. Colpitts

tmcol@bu.edu

${ }^{+}$Co-first authorship.

Specialty section:

This article was submitted

to Epidemiology,

a section of the journal

Frontiers in Public Health

Received: 25 January 2018

Accepted: 03 April 2018

Published: 18 May 2018

Citation:

Londono-Renteria BL, Shakeri H,

Rozo-Lopez P, Conway MJ,

Duggan N, Jaberi-Douraki M and Colpitts TM (2018) Serosurvey of

Human Antibodies Recognizing Aedes aegypti D7 Salivary Proteins in

Colombia.

Front. Public Health 6:111. doi: 10.3389/fpubh.2018.00111

\section{Berlin L. Londono-Renteria ${ }^{1 \dagger}$, Heman Shakeri't, Paula Rozo-Lopez ${ }^{1}$, Michael J. Conway ${ }^{3}$, Natasha Duggan ${ }^{4}$, Majid Jaberi-Douraki ${ }^{2,5 *}$ and Tonya M. Colpitts ${ }^{6 *}$}

${ }^{1}$ Department of Entomology, Kansas State University, Manhattan, KS, United States, ${ }^{2}$ Department of Anatomy and Physiology, Institute of Computational Comparative Medicine, Kansas State University, Manhattan, KS, United States, ${ }^{3}$ Central Michigan University College of Medicine, Mount Pleasant, MI, United States, ${ }^{4}$ Department of Cell Biology, University of Miami Miller School of Medicine, Miami, FL, United States, ${ }^{5}$ Department of Mathematics, Institute of Computational Comparative Medicine, Kansas State University, Manhattan, KS, United States, ${ }^{6}$ National Emerging Infectious Diseases Laboratories (NEIDL), Department of Microbiology, Boston University School of Medicine, Boston, MA, United States

Background: Dengue is one of the most geographically significant mosquito-borne viral diseases transmitted by Aedes mosquitoes. During blood feeding, mosquitoes deposit salivary proteins that induce antibody responses. These can be related to the intensity of exposure to bites. Some mosquito salivary proteins, such as D7 proteins, are known as potent allergens. The antibody response to D7 proteins can be used as a marker to evaluate the risk of exposure and disease transmission and provide critical information for understanding the dynamics of vector-host interactions.

Methods: The study was conducted at the Los Patios Hospital, Cucuta, Norte de Santander, Colombia. A total of 63 participants were enrolled in the study. Participants were categorized into three disease status groups, age groups, and socioeconomic strata. The level of IgG antibodies against D7 Aedes proteins was determined by ELISA. We used a statistical approach to determine if there is an association between antibody levels and factors such as age, living conditions, and dengue virus (DENV) infection.

Results: We found that IgG antibodies against D7 proteins were higher in non-DENV infected individuals in comparison to DENV-infected participants. Also, the age factor showed a significant positive correlation with IgG antibodies against D7 proteins, and the living conditions (socioeconomic stratification), in people aged 20 years or older, are a statistically significant factor in the variability of $\lg G$ antibodies against D7 proteins.

Conclusion: This pilot study represents the first approximation to elucidate any correlation between the antibody response against mosquito D7 salivary proteins and its correlation with age, living conditions, and DENV infection in a dengue endemic area.

Keywords: Aedes saliva, D7, antibody levels, biomarker, dengue

Abbreviations: DENV, dengue virus; D7S, protein D7 short; D7L, protein D7 long; SGE, salivary gland extract; $\triangle \mathrm{OD}$, delta optical density. 


\section{INTRODUCTION}

Dengue is one of the most globally significant mosquito-borne viral diseases, and epidemics are often recorded unevenly across socioeconomic strata (1). Tropical and subtropical areas of the Americas have shown a significant rise in dengue virus (DENV) transmission in recent years; especially in South America, where more than $80 \%$ of the population lives in areas of high risk (2). Historically, Colombia is one of the countries most affected by epidemics of dengue disease (3). Colombia has hyper-endemicity of multiple DENV serotypes and also has the presence of the two main transmission vector species: Aedes aegypti and Aedes albopictus $(3,4)$. While most DENV infections present little or no clinical symptoms, a small proportion of cases progresses to severe forms (1). The variability in clinical presentation of symptoms makes tracking DENV transmission rates difficult among susceptible populations (4). Dengue epidemics show local variations in risk influenced by the densities of mosquito-vector populations due to rainfall, temperature and unplanned rapid urbanization, suboptimal housing conditions, and poor access to clean water $(5,6)$. Recently, a high correlation has been reported between dengue transmission and the poorest areas belonging to the lowest levels of the socioeconomic stratification $(7,8)$.

During blood feeding, Aedes female mosquitoes inject saliva into host skin to facilitate blood uptake, which initiates a proteinmediated immune response (9). Previous research indicates that mosquito salivary proteins can induce significant antibody responses closely related to the number of bites received by a person. This can then be translated as the intensity of exposure, and could conceivably be used as markers to evaluate vector exposure and dengue transmission risk $(10,11)$. Certain salivary proteins are known as genus-specific biomarkers, which can help pinpoint vector exposure among host population (12). Specifically, the gSG6 and cE5 proteins are good indicators of human exposure to Anopheles mosquito bites while the Nterm- $34 \mathrm{kDa}$ peptide allows for evaluation of human exposure to Aedes bites $(13,14)$. Although human exposure does not always present disease transmission, human disease status impacts the antibody response to new exposures (12). Specifically, the D7 family of mosquito salivary proteins has been found to induce higher antibody levels depending on the disease status of the individual $(9,15)$. Previous research has also indicated that the $38 \mathrm{kDa}$ 7 protein is upregulated in mosquitoes infected with DENV2 (15). Moreover, the D7 immunomodulatory proteins deflect the vertebrate response against biting injury caused during mosquito feeding $(16,17)$ and potentially favor dengue transmission by recruiting target cells to the bite site $(9,18)$.

Although considerable research in DENV is focused on pathogen-induced disease severity (19-22), there is a need for studies focused on the role of arthropod vector factors, such as salivary proteins, on DENV infectivity and disease development (23). The objective of this study was to determine whether there is an association between antibody levels against two different Ae. aegypti D7 salivary proteins, D7 long (D7L) and D7 short (D7S), and DENV infection in people naturally exposed to $A e$. aegypti bites in dengue-endemic areas from Colombia. We also evaluated the association between the antibody response against the $\mathrm{D} 7$ proteins and factors such as age and living conditions.
The likelihood of blood feeding and dengue transmission increases as socioeconomic status decreases due to the proximity of human hosts to vector populations $(2,24,25)$. This pilot study represents the first approximation to elucidate any correlation between the antibody response against D7 salivary proteins and the distribution of such response in dengue-endemic areas.

\section{MATERIALS AND METHODS}

\section{Study Design and Population}

The protocols and methods for this study were reviewed and approved by Los Patios Hospital Ethics Review Board (FGI01-06). Participant's recruitment was conducted at the Los Patios Hospital (Norte de Santander-Colombia). Patients consulting at the Los Patios hospital for febrile illnesses compatible with dengue were included in the study. In brief, a patient with a "probable dengue" diagnosis and remitted to the laboratory for blood testing is invited to participate. As non-febrile controls, we enrolled non-febrile volunteers accompanying the febrile patients to the laboratory testing if the patient has already agreed to participate in the study. The research objective was explained to each participant and a written informed consent was obtained before collecting samples. A total of 63 participants were enrolled in the study from March to June of 2015. Participants were categorized into three disease status categories: status 1, non-febrile subjects, defined as companions of febrile patients, also considered as dengue exposed subjects but with a negative RT-PCR test for DENV. Status 2, febrile subjects, defined as febrile patients with compatible symptoms to dengue fever but with a negative RT-PCR test for DENV. Status 3, DENV infected subjects, defined as participants with a positive DENV infection confirmed by RT-PCR. DENV infection status was determined by the presence of viral RNA extracted from serum samples according to methods described by Londono-Renteria et al. (10) using DENV serotypes specific primers described elsewhere (26). Briefly, RNA was extracted from the participant's serum sample using the Quick RNAViral kit (Zymo Research). qRT-PCR conditions on the CFX96 Touch ${ }^{\mathrm{TM}}$ Real-Time PCR Detection System were: RT Step: $48^{\circ} \mathrm{C}$ for $5 \mathrm{~min}$, and $95^{\circ} \mathrm{C}$ for $2 \mathrm{~min}$. Amplification step: $\left(95^{\circ} \mathrm{C}\right.$ for $15 \mathrm{~s}$, $60^{\circ} \mathrm{C}$ for $\left.20 \mathrm{~s}\right) \times 40$ cycles.

The living conditions of participants are based on the socioeconomic stratification for the Barrios (Neighborhoods) in the city (Cucuta) according to the Colombian National standards (27). In Colombia, the socioeconomic stratification is divided into six categories, with stratum no. 1 as the lowest and stratum no. 6, the highest. The stratification is based on urban settings characteristics, housing conditions, and access to public utilities (27). In this study, the living conditions of the participants corresponded to three lowest types of strata according to the Colombia's socioeconomic stratification: Stratum 1 as lower-low, stratum 2 as low, and stratum 3 as upper-low. There was no participant in this study that reported living in any of the higher strata (Stratum 4 or median, stratum 5 or median-high, and stratum 6 or high).

\section{D7 Recombinant Proteins}

The recombinant D7L protein (AAEL006424) NCBI accession \#EAT41994, UniProtKB/Swiss-Prot: P18153.2 and the D7S 
protein [UniProtKB-Q1HRR6 (Q1HRR6_AEDAE)] were produced according to the methods published by Conway et al. (15). Protein concentration was determined using the Thermo Scientific NanoDrop ${ }^{\mathrm{TM}}$ (Thermo Fisher Scientific, Wilmington, DE, USA). All ELISA testing was performed using $1 \mu \mathrm{g} / \mathrm{ml}$ as the final protein concentration.

\section{Antibody Detection by ELISA}

The level of human IgG antibodies against mosquito salivary proteins was determined by an indirect ELISA following the methods published by Londono-Renteria et al. $(10,28)$. Briefly, 96-well ELISA plates (Nunc-Maxisorp, Nalgene Nunc International, Rochester, NY, USA) were coated with $100 \mu \mathrm{l} /$ well of Ae. aegypti salivary gland extract (SGE) or individual D7 recombinant proteins in a final concentration of $0.5 \mu \mathrm{g} / \mathrm{ml}$ of prepared in coating solution (Kierkegaard and Perry Laboratories, Gaithersburg, MD, USA). Serum samples were tested in duplicate in a $1 / 100$ dilution. After washes, plates were incubated with horseradish peroxidase-conjugated goat anti-human IgG $(1: 1,000)$ (Abcam, Ab81202) and colorimetric development was obtained using tetra-methyl-benzidine (one-solution micro-well, Gene-Script, Piscataway, NJ, USA). The reaction was stopped with $1 \mathrm{M}$ phosphoric acid and absorbance was measured at $450 \mathrm{~nm}$. Three controls were included on each plate: (1) control blank: two wells without SGE as control for nonspecific induction of color for any of the reagents used in the test; (2) negative control: two wells with SGE but without human serum as control for any nonspecific color induction of the coating antigen; and (3) positive control: 1 control per plate to test plate variation and normalize OD (optical density) values. IgG antibody levels are reported as $\Delta \mathrm{OD}=$ Average patient $\mathrm{OD}$ value (duplicate) - Blank OD value (28).

\section{Statistical Analysis}

Differences in the antibody levels $(\Delta \mathrm{OD})$ between two groups (i.e., IgG levels in non-febrile subjects versus IgG levels in DENV infected subjects) were measured by the non-parametrical
Mann-Whitney $U$ test; while Spearman rank's correlation test was used to measure association between variables within and between groups (i.e., age and IgG antibody levels). We also conducted a three-way analysis of variance (ANOVA) to compare the main and partial influences of age, disease status, and living conditions (as socioeconomic stratification) on the IgG antibodies against D7S and D7L proteins.

Cluster analysis of data using $k$-mean method, suggests categorizing the participants roughly into four age groups: group 1, ages 1-19 years old; group 2, ages 20-39 years old; group 3, ages 40-59 years old; and group 4 with individuals older than 60 years. In addition to determining whether a factor has a statistically significant difference in the mean level of IgG (D7L or D7S), we measured the effect size of such factor by partial eta-squared. These statistics explain the proportion of variance accounted by an independent effect. Partial etasquared shows better consistency than eta-squared for measuring effect size in experiments with different designs (29). The effect size was obtained from and standardized according to Richardson et al. (30), and the following guidelines from Ref. (29):

- $\eta^{2}<0.059$ represents a small effect size

- $0.059 \leq \eta^{2}<0.138$ represents a medium effect size

- $\eta^{2} \geq 0.138$ represents a large effect size.

Statistical analyses were performed using R 3.4.

\section{RESULTS}

Sixty-three volunteers were included in this pilot study ( 28 females and 35 males) with an age range between 1 and 82 years old (mean age $=22.6$ ). The analysis of the antibody response against the D7 proteins and the disease status (non-febrile, febrile, and dengue positive) indicates a higher antibody response against the D7S in non-febrile subjects in comparison to DENV-infected subjects ( $p=0.0742)$ while non-febrile subjects presented slightly lower antibody levels against the D7L than the other groups $(p=0.3435)$ (Figure 1).
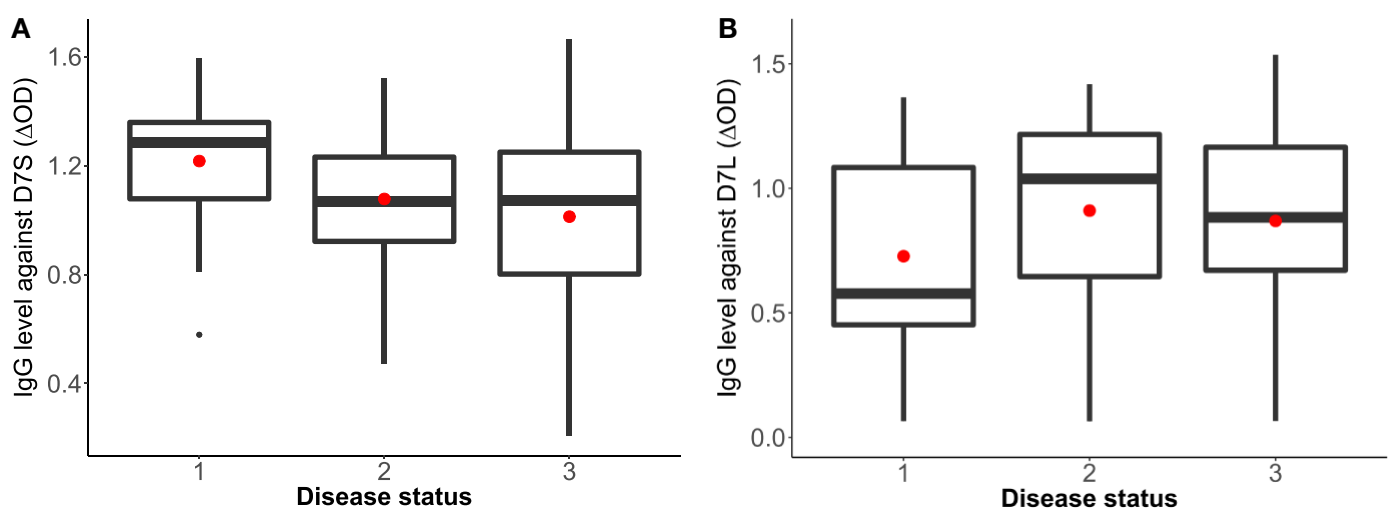

FIGURE 1 | Human IgG antibody levels against Aedes aegypti D7 short (A) and D7 long (B) salivary proteins and disease status. Status 1: non-febrile, non-dengue virus (DENV) infected control subjects $(n=12)$, status 2 febrile subjects $(n=12)$, and status 3: DENV infected subjects $(n=37)$. The red dot indicates the mean and the bars show the first and third quartiles with median as the centerline. 
Our analysis of the antibody levels by age categories revealed a significant positive correlation between age and IgG antibodies against D7S $\left(r^{2}=0.3820, p=0.0024\right)$ (Figure 2). We also observed a positive correlation between age and IgG antibodies against D7L; however, this association was not statistically significant $\left(r^{2}=0.1067, p=0.4132\right)$.

Table 1 shows the results of three analyses with the test statistics $F$-ratio, the statistical significance with $\alpha=0.05$ and also effect size on the analysis. In order to ease the reading of the table, Cohen's guidelines for the effect size are included (29). The main effect for age yielded an $F$-ratio of $F(3,53)=4.685$ and $p=0.005$, demonstrating a significant difference between observed means of IgG concentration against D7S among the different age groups. A pairwise $t$-test (with pooled variance) suggested a significant difference between age group 1 (1-19 years old) and the rest of the age groups. This result indicates that the average of individuals in age group 1 exhibits significantly different IgG antibody level against D7S, also observed in Figure 2.

To isolate the effects of age group 1 in the IgG antibody level against D7S, we excluded individuals under 20 years old and repeated the analysis (Table 2 ). Furthermore, when age group 1 was excluded, we found that living conditions (socioeconomic stratification) are a statistically significant factor $(\alpha=0.045)$ in the variability of levels of IgG against D7S. The analysis of the antibody response against the D7S protein for individuals older than 20 years showed higher antibody levels in participants living in stratum 2 (low living conditions) (Figure 3). D7L antibody levels show similarity across the evaluated three socioeconomic strata.

Our analysis also revealed significantly higher IgG antibody levels in non-febrile patients living in strata 1 and 2 against D7S
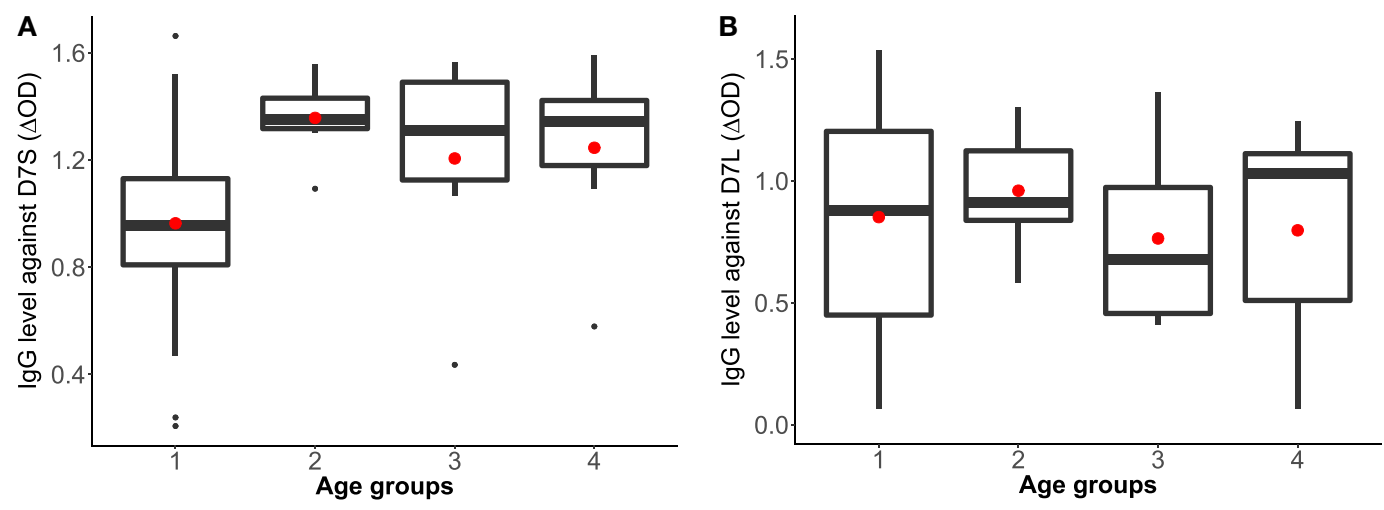

FIGURE 2 | Aedes aegypti D7 short (A) and D7 long (B) salivary proteins and lgG antibody levels represented by age groups. Group 1: ages 1-19 years old $(n=41)$, group 2: ages 20-39 years old $(n=7)$, group 3: ages 40-59 years old $(n=6)$; and group 4 : individuals older than 60 years $(n=7)$. The red dot indicates the mean and the bars show the first and third quartiles with median as the centerline.

TABLE 1 | Analysis of variance table showing the test statistics $F$-ratio, statistical significance with $\alpha=0.05$, and effect size reported for each variable (age, disease status, and living conditions) evaluated in D7 short and D7 Long for the total sample size ( $n=63$ participants).

\begin{tabular}{|c|c|c|c|c|c|}
\hline D7 & Effect & $\begin{array}{c}\text { F-ratio } \\
\text { (df1,df2) }\end{array}$ & Statistically significant ( $p$-value) & eta-squared & Effect size (Cohen's guidelines) \\
\hline \multirow[t]{3}{*}{ D7 short } & Age & $4.685(3,53)$ & Yes $(0.005)$ & 0.168 & Large \\
\hline & Disease status & $0.450(2,53)$ & No $(0.634)$ & 0.014 & Small \\
\hline & Socioeconomic strata & $0.337(2,53)$ & No $(0.710)$ & 0.012 & Small \\
\hline \multirow[t]{3}{*}{ D7 long } & Age & $0.295(3,53)$ & No (0.829) & 0.022 & Small \\
\hline & Disease status & $0.747(2,53)$ & No (0.479) & 0.038 & Small \\
\hline & Socioeconomic strata & $1.149(2,53)$ & No (0.325) & 0.041 & Small \\
\hline
\end{tabular}

The numerator and denominator degrees of freedom is shown by df1 and df2.

TABLE 2 | Analysis of variance table showing the test statistics $F$-ratio, statistical significance with $\alpha=0.05$, and effect size reported for each variable (age, disease status, and living conditions) evaluated in D7 short excluding age group 1 (participants under 20 years old).

\begin{tabular}{|c|c|c|c|c|c|}
\hline & Treatment & $\begin{array}{c}\text { F-ratio } \\
\text { (df1,df2) }\end{array}$ & Statistically significant ( $p$-value) & eta-squared & Effect size (Cohen's guidelines) \\
\hline \multirow[t]{3}{*}{ D7 short (age > 20) } & Age & $0.523(2,10)$ & No $(0.538)$ & 0.0151 & Small \\
\hline & Disease status & $0.193(2,10)$ & No $(0.788)$ & 0.094 & Medium \\
\hline & Socioeconomic strata & $3.975(2,10)$ & Yes $(0.031)$ & 0.379 & Large \\
\hline
\end{tabular}

The numerator and denominator degrees of freedom is shown by df1 and df2. 

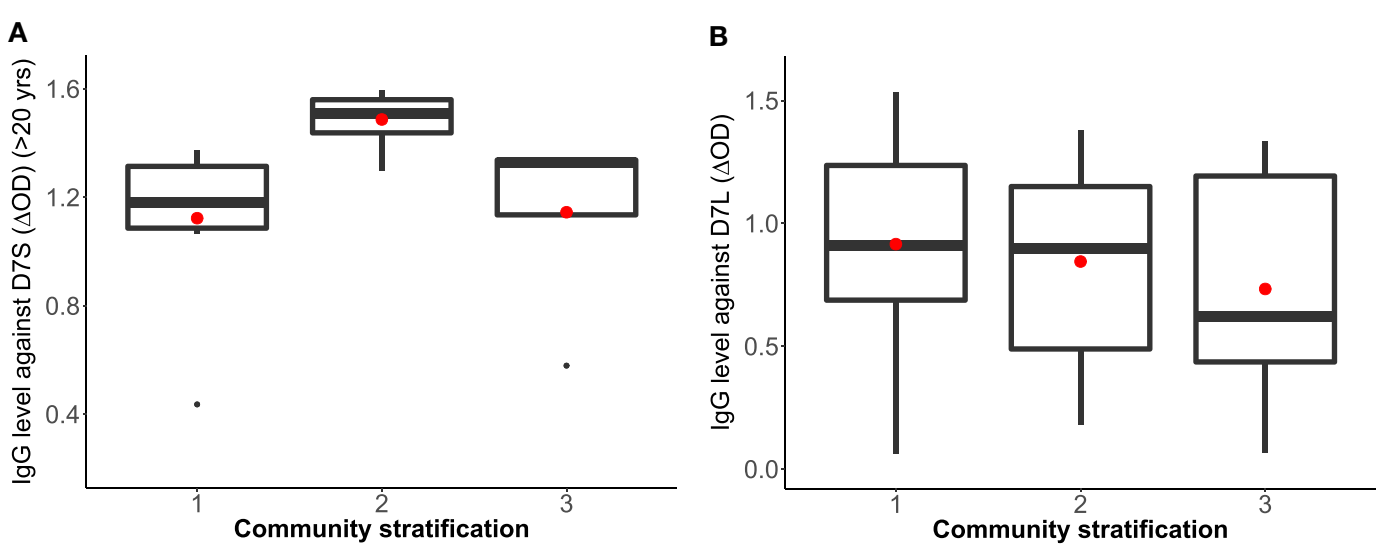

FIGURE 3 | Correlation analysis between Aedes aegypti D7 short (A) and D7 long (B) salivary proteins and living conditions represented as socioeconomic stratification. Stratum 1: lower-low conditions (D7S $n=8, \mathrm{D} 7 \mathrm{~L} n=23)$, stratum 2: low conditions (D7S $n=8$, D7L $n=26)$, and stratum 3: upper-low conditions (D7S $n=4$, D7L $n=12$ ). Notice that D7S graphic excludes participants under 20 years old in the analysis.
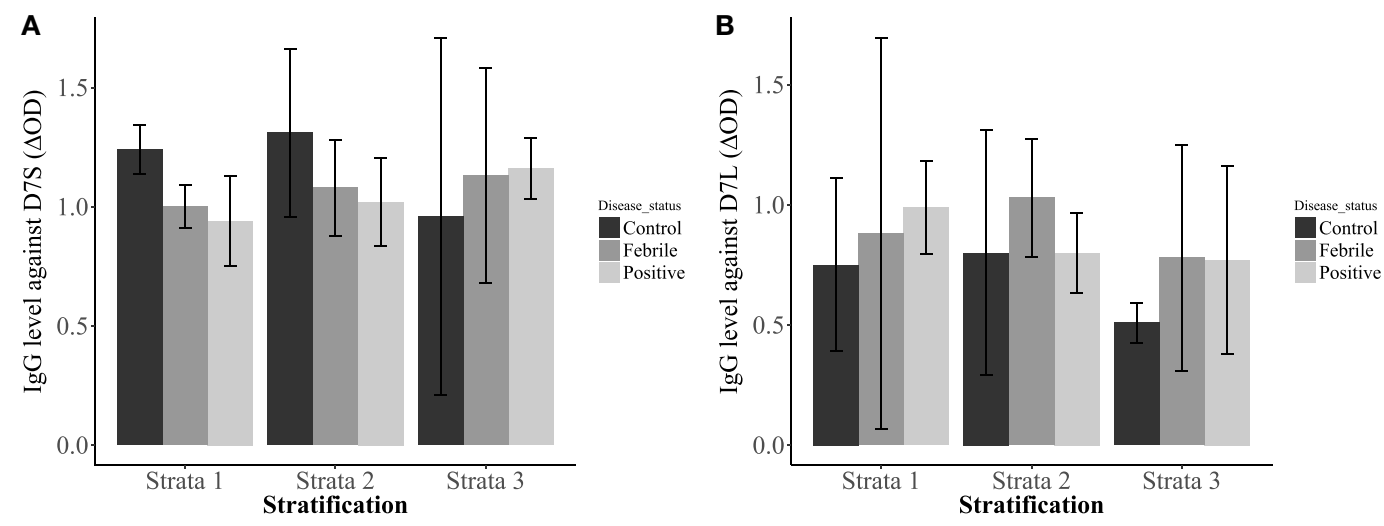

FIGURE 4 | Interaction plots showing the concentrations of lgG antibodies against D7 short (A) and D7 long (B) when evaluated against the patient disease status: non-febrile subjects (control) $(n=12)$, febrile subjects $(n=12)$, and dengue virus positive subjects $(n=37)$ and living conditions [stratum 1 : lower-low conditions ( $n=23)$, stratum 2: low conditions $(n=26)$, and stratum 3: upper-low conditions $(n=12)]$.

(Figure 4A). However, the socioeconomic stratum 3 displayed higher IgG antibodies against D7S for febrile and DENV positive participants. Although none of the factors evaluated for D7L showed statistical significance, the effects of disease status and living conditions are higher than age effects (Table 1). Our tests also revealed that D7L is generally higher in febrile patients than non-febrile subjects (control status) (Figure 4B).

\section{DISCUSSION}

Models incorporating anti-salivary protein antibody levels have been shown to be powerful and sensitive biomarkers for a direct and accurate evaluation of the human exposure to mosquito bites (12, 31, 32). Moreover, the detection of increased levels of anti-vector saliva could be pointed as an epidemiological marker of infection and also a suitable indicator of clinical immunity in endemic regions (12). In the case of dengue, it has been shown that antibodies against SGE of Ae. aegypti can work successfully as markers of mosquito exposure $(12,33,34)$. Furthermore, our previous studies indicate that in dengue-endemic regions, individuals infected with DENV had significantly higher antibody titers to total Aedes SGE than non-DENV infected patients $(10,35)$. Although the whole SGE has been widely used as an approximation to evaluate exposure, SGE enhances the probability of cross-reactivity that may impair the evaluation of the exposure to different vector species (12). This cross-reactivity is caused by different antigens, such as conserved mosquito salivary components, which appear similar to the host molecules or to other arthropod vectors of human disease (36-38). Therefore, the use of species-specific proteins will allow to better calculate dengue transmission and Aedes-human contact dynamics. Thus, the finding of a potential "infective bite" marker would significantly advance the protocols available to study the epidemiology of vector-borne febrile illness exposure and help in the design of recommendation guidelines to travelers around the world.

The mosquito D7 salivary proteins were shown to bind biogenic amines, which are important mediators of inflammation, allergy 
and vascular permeability, and vascular tone (39); consequently, D7 proteins were identified as promising biomarkers of exposure to Aedes bites $(33,34)$. Several members of the $\mathrm{D} 7$ protein family are upregulated in DENV infected mosquitoes, and when directly interact with virions, cause infection inhibition $(15,40)$; however, previous study suggests that, in the human host, the presence of anti-D7 antibodies may be linked to enhanced disease severity (35).

Our analysis showed higher IgG levels against D7L in DENV positive and febrile patients as compared to non-febrile subjects. Previous studies demonstrate that individuals who are exposed to mosquitoes have higher levels of anti-D7L antibodies and the presence of anti-D7 antibodies has been linked to disease severity $(15,35,40)$. Conversely, anti-D7 antibodies may prevent mosquitoes from efficient blood feeding, which may enhance disease transmission and disease severity (15). Since D7L is upregulated in infected mosquitoes, our results are consistent with showing that high salivary protein concentrations correlate to higher induction of antibodies in the human host. Thus, the characterizing the factors involved in the vector saliva-virus-host interactions will lead to the development of better strategies to limit disease transmission. The small sample size is one limitation of the current study and larger studies will be necessary to fully understand the role of $\mathrm{D} 7 \mathrm{~L}$ antibodies and their potential as biomarkers for risk of DENV infection.

Historically, the highest incidence of dengue disease in Colombia was in individuals between 15 and 44 years of age (7). However, in the last decade, the highest rates of occurrence for dengue infections have shifted to younger populations from 4 to 14 years of age (3). Our results indicate that the IgG response to D7S was lower in young populations (under 19 years of age). There was a large effect size in the age variable and a substantial difference in the sample size between age group 1 and the other three groups. Our research further suggests that anti-D7 IgG response can separate both the adaptive condition of exposures in the older population and recent exposure with increased antibody titers in younger populations.

In Colombia, a socioeconomic stratification is a defined classification of the residential real estate by household conditions, which are reflected on differential charges of domiciliary utilities. The classification in any of the six strata is an approximation to the hierarchical socioeconomic difference, from poverty $(1,2,3)$ to wealth $(4,5,6)$ (DANE-Colombia). In this way, those who have more economic capacity will pay more for public services and contribute so that the lower strata can obtain the services at affordable rates (DANE-Colombia). As a result of this classification system, in Colombia, it is possible to measure the impact to society from shifts in population densities, political, and economic policies as well hazards to human health such as those posed by vector-borne diseases. In the case of dengue, it has been shown that the spatial distribution and impact of the disease are influenced by environmental and socioeconomic factors such as education, occupation, income, population density, and livelihoods (7, 8, 25, 41, 42). Urban space and housing settings are also important dimensions to impacting the dynamics of dengue outbreaks (42). However, the socioeconomic variables seem to be context-dependent, scale-specific, and mainly driven by data availability (7). Studies in Thailand (42), Saudi Arabia (43), and Brazil $(24,25)$ showed a strong positive association between dengue fever cases and socioeconomic factors. Those studies reveal that the prevalence of dengue infection is significantly higher in deprived areas (lower strata) in comparison with intermediate and privileged areas (upper strata). Therefore, dengue risk is inversely related to the socioeconomic status. Moreover, in deprived areas, the majority of children had already been exposed to DENV by the age of 5 years $(4,24,25,41,42)$. Although, in this study, we only included the three lower socioeconomic strata because there are historically more associated with the presence of dengue, we were expecting a clear negative correlation between the IgG antibody levels and the socioeconomic status. However, we see that the antibody levels were significantly higher in the strata 2 , although we still do not know the exact reason for this result, we believe it is possible that a confounder causes. One of the factors that may be influencing the results is the way stratification is characterized in the area. For instance, previous studies in Colombia have found heterogeneity in dengue exposure and disease within houses sharing the same stratification (7). In addition, house construction materials, access to the sewage system and vegetation coverage, factors not included within the stratification classification, can differ between houses within the same strata. Also, the presence of plastics or other breeding sites for mosquitoes around the houses may influence (11). Unfortunately, we did collect any individual information on the houses, so we cannot exactly pinpoint where the confounder may be. A larger study where the individual household conditions for each study subject may help to clarify these findings.

The investigation into sociodemographic perspective can provide insights for effective management and prevention approaches in areas of high dengue transmission risk (8); however, data-driven tools must incorporate models, which address the physiology of transmission to better estimate transmission risk $(44,45)$. The use of biomarkers such as the antibodies against salivary proteins of relevance in virus replication is important to identify critical areas within endemic regions where interventions may have higher impact interrupting virus transmission.

In order to evaluate whether socioeconomic stratifications are significant predictors of DENV risk at an urban scale, further research is needed to explore the presence of other salivary biomarkers. Establishing a database of specific biomarkers that relates DENV risk to socioeconomic factors will provide support for public health strategies to enable better detection in high-risk areas. Our research suggests that identifying an Aedes-specific salivary protein as an infective bites biomarker would help to more efficiently identify individuals who have been in contact with an infected vector and, therefore, would help to more accurately evaluate the risk of disease transmission.

\section{CONCLUSION}

Mosquito saliva plays an important role in vector-borne disease transmission and pathology. Immune responses induced by salivary proteins may also have a profound impact on the clinical presentation of diseases like dengue. The present study is a first step toward being able to use human IgG responses to D7 salivary proteins as a biomarker for exposure to infective Aedes bites. We found that age is a significant factor in the level of D7S 
and pooling data for all age groups eliminates effects of patient status and living conditions. Therefore, by separating individuals by their ages, significant large effect for living condition (living strata) and a medium effect, although non-significant, of patient status were observed. However, further studies are needed to test the sensitivity of the biomarker in epidemic settings where Ae. aegypti-borne diseases are emerging or re-emerging. A better understanding of the interactions between the host, the vector, and the virus will be valuable to designing more accurate guidelines in endemic regions where residents are frequently bitten by both uninfected and infected mosquitoes.

\section{CONSENT FOR PUBLICATION}

The authors have no affiliations that represent a conflict of interest regarding this manuscript.

\section{AVAILABILITY OF DATA AND MATERIALS}

The datasets used and/or analyzed during the current study are available from the corresponding author upon reasonable request.

\section{ETHICS STATEMENT}

Protocols and research methods were reviewed and approved by Universidad de Pamplona and the Ethics Review Board of Hospital
Erasmo Meoz. The investigation was explained to each individual, and a written informed consent was obtained from each participant or their legal guardian before collecting samples. Blood samples were collected in compliance with the regulations on ethics of research in human participants for Colombia and the United States.

\section{AUTHOR CONTRIBUTIONS}

BL-R: experimental design and manuscript writing. MC, ND, and TC: recombinant protein synthesis. HS and MJ-D: experimental design, statistical analysis, and manuscript writing. PR-L and TC: manuscript writing.

\section{ACKNOWLEDGMENTS}

This work was in collaboration with Boston University, Tulane University, and Los Patios Hospital_Colombia. We would like to thank Jenny Carolina Cardenas for her support in this research and the Norte de Santander Community for always supporting our work. This work was also supported by Kansas Bioscience Authority via their support for the Institute of Computational Comparative Medicine at Kansas State University.

\section{FUNDING}

TC was funded by NIH. This work was funded in part by: TCK22 Al103067-01, BL-R-NIFA-NE1443.

\section{REFERENCES}

1. Torres JR, Orduna TA, Pina-Pozas M, Vazquez-Vega D, Sarti E. Epidemiological characteristics of dengue disease in Latin America and in the Caribbean: a systematic review of the literature. J Trop Med (2017) 2017:8045435. doi:10.1155/2017/8045435

2. Ramos-Castaneda J, Barreto Dos Santos F, Martinez-Vega R, Galvao de Araujo JM, Joint G, Sarti E. Dengue in Latin America: systematic review of molecular epidemiological trends. PLoS Negl Trop Dis (2017) 11(1):e0005224. doi:10.1371/journal.pntd.0005224

3. Villar LA, Rojas DP, Besada-Lombana S, Sarti E. Epidemiological trends of dengue disease in Colombia (2000-2011): a systematic review. PLoS Negl Trop Dis (2015) 9(3):e0003499. doi:10.1371/journal.pntd.0003499

4. Lee JS, Mogasale V, Lim JK, Carabali M, Lee KS, Sirivichayakul C, et al. A multi-country study of the economic burden of dengue fever: Vietnam, Thailand, and Colombia. PLoS Negl Trop Dis (2017) 11(10):e0006037. doi:10.1371/journal.pntd.0006037

5. Bowman LR, Runge-Ranzinger S, McCall PJ. Assessing the relationship between vector indices and dengue transmission: a systematic review of the evidence. PLoS Negl Trop Dis (2014) 8(5):e2848. doi:10.1371/journal.pntd.0002848

6. Xu L, Stige LC, Chan KS, Zhou J, Yang J, Sang S, et al. Climate variation drives dengue dynamics. Proc Natl Acad Sci U S A (2017) 114(1):113-8. doi:10.1073/ pnas. 1618558114

7. Delmelle E, Hagenlocher M, Kienberger S, Casas I. A spatial model of socioeconomic and environmental determinants of dengue fever in Cali, Colombia. Acta Trop (2016) 164:169-76. doi:10.1016/j.actatropica.2016.08.028

8. Hagenlocher M, Delmelle E, Casas I, Kienberger S. Assessing socioeconomic vulnerability to dengue fever in Cali, Colombia: statistical vs expert-based modeling. Int J Health Geogr (2013) 12:36. doi:10.1186/1476-072X-12-36

9. Pingen M, Bryden SR, Pondeville E, Schnettler E, Kohl A, Merits A, et al. Host inflammatory response to mosquito bites enhances the severity of arbovirus infection. Immunity (2016) 44(6):1455-69. doi:10.1016/j.immuni.2016. 06.002

10. Londono-Renteria B, Cardenas JC, Cardenas LD, Christofferson RC, Chisenhall DM, Wesson DM, et al. Use of anti-Aedes aegypti salivary extract antibody concentration to correlate risk of vector exposure and dengue transmission risk in Colombia. PLoS One (2013) 8(12):e81211. doi:10.1371/ journal.pone.0081211

11. Londono-Renteria B, Cardenas JC, Giovanni JE, Cardenas L, Villamizar P, Rolon J, et al. Aedes aegypti anti-salivary gland antibody concentration and dengue virus exposure history in healthy individuals living in an endemic area in Colombia. Biomedica (2015) 35(4):572-81. doi:10.7705/biomedica. v35i4.2530

12. Doucoure S, Drame PM. Salivary biomarkers in the control of mosquito-borne diseases. Insects (2015) 6(4):961-76. doi:10.3390/insects6040961

13. Rizzo C, Lombardo F, Ronca R, Mangano V, Sirima SB, Nebie I, et al. Differential antibody response to the Anopheles gambiae gSG6 and cE5 salivary proteins in individuals naturally exposed to bites of malaria vectors. Parasit Vectors (2014) 7:549. doi:10.1186/s13071-014-0549-8

14. Ndille EE, Dubot-Peres A, Doucoure S, Mouchet F, Cornelie S, Sidavong B, et al. Human IgG antibody response to Aedes aegypti Nterm-34 kDa salivary peptide as an indicator to identify areas at high risk for dengue transmission: a retrospective study in urban settings of Vientiane city, Lao PDR. Trop Med Int Health (2014) 19(5):576-80. doi:10.1111/tmi.12280

15. Conway MJ, Londono-Renteria B, Troupin A, Watson AM, Klimstra WB, Fikrig E, et al. Aedes aegypti D7 saliva protein inhibits dengue virus infection. PLoS Negl Trop Dis (2016) 10(9):e0004941. doi:10.1371/journal.pntd.0004941

16. Fontaine A, Diouf I, Bakkali N, Misse D, Pages F, Fusai T, et al. Implication of haematophagous arthropod salivary proteins in host-vector interactions. Parasit Vectors (2011) 4:187. doi:10.1186/1756-3305-4-187

17. Leitner WW, Costero-Saint Denis A, Wali T. Immunological consequences of arthropod vector-derived salivary factors. Eur J Immunol (2011) 41(12): 3396-400. doi:10.1002/eji.201190075

18. Bizzarro B, Barros MS, Maciel C, Gueroni DI, Lino CN, Campopiano J, et al. Effects of Aedes aegypti salivary components on dendritic cell and lymphocyte biology. Parasit Vectors (2013) 6:329. doi:10.1186/1756-3305-6-329 
19. Kuhn RJ, Dowd KA, Beth Post C, Pierson TC. Shake, rattle, and roll: impact of the dynamics of flavivirus particles on their interactions with the host. Virology (2015) 479-480:508-17. doi:10.1016/j.virol.2015.03.025

20. Schmid MA, Diamond MS, Harris E. Dendritic cells in dengue virus infection: targets of virus replication and mediators of immunity. Front Immunol (2014) 5:647. doi:10.3389/fimmu.2014.00647

21. Green AM, Beatty PR, Hadjilaou A, Harris E. Innate immunity to dengue virus infection and subversion of antiviral responses. J Mol Biol (2014) 426(6):1148-60. doi:10.1016/j.jmb.2013.11.023

22. Morrison J, Aguirre S, Fernandez-Sesma A. Innate immunity evasion by dengue virus. Viruses (2012) 4(3):397-413. doi:10.3390/v4030397

23. Cox J, Mota J, Sukupolvi-Petty S, Diamond MS, Rico-Hesse R. Mosquito bite delivery of dengue virus enhances immunogenicity and pathogenesis in humanized mice. J Virol (2012) 86(14):7637-49. doi:10.1128/JVI.00534-12

24. Braga C, Luna CF, Martelli CM, de Souza WV, Cordeiro MT, Alexander N, et al. Seroprevalence and risk factors for dengue infection in socio-economically distinct areas of Recife, Brazil. Acta Trop (2010) 113(3):234-40. doi:10.1016/j. actatropica.2009.10.021

25. Mondini A, Chiaravalloti-Neto F. Spatial correlation of incidence of dengue with socioeconomic, demographic and environmental variables in a Brazilian city. Sci Total Environ (2008) 393(2-3):241-8. doi:10.1016/j. scitotenv.2008.01.010

26. Johnson BW, Russell BJ, Lanciotti RS. Serotype-specific detection of dengue viruses in a fourplex real-time reverse transcriptase PCR assay. J Clin Microbiol (2005) 43(10):4977-83. doi:10.1128/JCM.43.10.4977-4983.2005

27. Estratificaciónsocioeconómica.Availablefrom:http://www.dane.gov.co/index. $\mathrm{php} /$ servicios-al-ciudadano/servicios-de-informacion/estratificacionsocioeconomica

28. Londono-Renteria B, Drame PM, Weitzel T, Rosas R, Gripping C, Cardenas JC, et al. An. gambiae gSG6-P1 evaluation as a proxy for human-vector contact in the Americas: a pilot study. Parasit Vectors (2015) 8:533. doi:10.1186/ s13071-015-1160-3

29. Cohen J. The statistical power of abnormal-social psychological research: a review. J Abnorm Soc Psychol (1962) 65:145-53. doi:10.1037/h0045186

30. Richardson JTE. Eta squared and partial eta squared as measures of effect size in educational research. Educ Res Rev (2011) 6(2):135-47. doi:10.1016/j. edurev.2010.12.001

31. Doucoure S, Cornelie S, Patramool S, Mouchet F, Demettre E, Seveno M, et al. First screening of Aedes albopictus immunogenic salivary proteins. Insect Mol Biol (2013) 22(4):411-23. doi:10.1111/imb.12032

32. Doucoure S, Mouchet F, Cornelie S, Drame PM, D’Ortenzio E, DeHecq JS, et al. Human antibody response to Aedes albopictus salivary proteins: a potential biomarker to evaluate the efficacy of vector control in an area of chikungunya and dengue virus transmission. Biomed Res Int (2014) 2014:746509. doi:10.1155/2014/746509

33. Doucoure S, Mouchet F, Cournil A, Le Goff G, Cornelie S, Roca Y, et al. Human antibody response to Aedes aegypti saliva in an urban population in Bolivia: a new biomarker of exposure to dengue vector bites. Am J Trop Med Hyg (2012) 87(3):504-10. doi:10.4269/ajtmh.2012.11-0477

34. Coutinho-Abreu IV, Guimaraes-Costa AB, Valenzuela JG. Impact of insect salivary proteins in blood feeding, host immunity, disease, and in the development of biomarkers for vector exposure. Curr Opin Insect Sci (2015) 10:98-103. doi:10.1016/j.cois.2015.04.014
35. Machain-Williams C, Mammen MP Jr, Zeidner NS, Beaty BJ, Prenni JE, Nisalak A, et al. Association of human immune response to Aedes aegypti salivary proteins with dengue disease severity. Parasite Immunol (2012) 34(1):15-22. doi:10.1111/j.1365-3024.2011.01339.x

36. Champagne DE, Ribeiro JM. Sialokinin I and II: vasodilatory tachykinins from the yellow fever mosquito Aedes aegypti. Proc Natl Acad Sci U S A (1994) 91(1):138-42. doi:10.1073/pnas.91.1.138

37. Rizzo C, Ronca R, Fiorentino G, Mangano VD, Sirima SB, Nebie I, et al. Wide cross-reactivity between Anopheles gambiae and Anopheles funestus SG6 salivary proteins supports exploitation of gSG6 as a marker of human exposure to major malaria vectors in tropical Africa. Malar J (2011) 10:206. doi:10.1186/1475-2875-10-206

38. Reagan KL, Machain-Williams C, Wang T, Blair CD. Immunization of mice with recombinant mosquito salivary protein $\mathrm{D} 7$ enhances mortality from subsequent West Nile virus infection via mosquito bite. PLoS Negl Trop Dis (2012) 6(12):e1935. doi:10.1371/journal.pntd.0001935

39. Calvo E, Mans BJ, Andersen JF, Ribeiro JM. Function and evolution of a mosquito salivary protein family. J Biol Chem (2006) 281(4):1935-42. doi:10.1074/ jbc.M510359200

40. Chisenhall DM, Londono BL, Christofferson RC, McCracken MK, Mores CN. Effect of dengue-2 virus infection on protein expression in the salivary glands of Aedes aegypti mosquitoes. Am J Trop Med Hyg (2014) 90(3):431-7. doi:10.4269/ajtmh.13-0412

41. Pastrana ME, Brito RL, Nicolino RR, de Oliveira CS, Haddad JP. Spatial and statistical methodologies to determine the distribution of dengue in Brazilian municipalities and relate incidence with the health vulnerability index. Spat Spatiotemporal Epidemiol (2014) 11:143-51. doi:10.1016/j.sste.2014. 04.001

42. Koyadun S, Butraporn P, Kittayapong P. Ecologic and sociodemographic risk determinants for dengue transmission in urban areas in Thailand. Interdiscip Perspect Infect Dis (2012) 2012:907494. doi:10.1155/2012/907494

43. Khormi HM, Kumar L. Modeling dengue fever risk based on socioeconomic parameters, nationality and age groups: GIS and remote sensing based case study. Sci Total Environ (2011) 409(22):4713-9. doi:10.1016/ j.scitotenv.2011.08.028

44. Carabali M, Ocampo CB, Toledo ME, Osorio L. [Mass communication of dengue surveillance data: effect of an intervention in Guadalajara de Buga, Colombia]. Biomedica (2013) 33(Suppl 1):130-41.

45. Gemperli A, Vounatsou P, Sogoba N, Smith T. Malaria mapping using transmission models: application to survey data from Mali. Am J Epidemiol (2006) 163(3):289-97. doi:10.1093/aje/kwj026

Conflict of Interest Statement: The authors declare that the research was conducted in the absence of any commercial or financial relationships that could be construed as a potential conflict of interest.

Copyright (c) 2018 Londono-Renteria, Shakeri, Rozo-Lopez, Conway, Duggan, Jaberi-Douraki and Colpitts. This is an open-access article distributed under the terms of the Creative Commons Attribution License (CC BY). The use, distribution or reproduction in other forums is permitted, provided the original author(s) and the copyright owner are credited and that the original publication in this journal is cited, in accordance with accepted academic practice. No use, distribution or reproduction is permitted which does not comply with these terms. 